International Journal of Bifurcation and Chaos, Vol. 29, No. 7 (2019) 1950087 [1] pages)

(C) The Author(s)

DOI: $10.1142 / \mathrm{S} 0218127419500871$

\title{
A Novel Algorithm for Detection and Localization of Periodic Phenomena of Chaotic Binary Sequences
}

\author{
Chunlei Fan*, Chuanfu Wang ${ }^{\dagger}$ and Qun Ding ${ }^{\ddagger}$ \\ Electrical Engineering College, Heilongjiang University, \\ Harbin 150080, P. R. China \\ *Chunlei_fan@aliyun.com \\ †chuanfuwang@aliyun.com \\ ‡qunding@aliyun.com
}

Received April 25, 2018; Revised July 15, 2018

\begin{abstract}
At present, the performance of the digital chaotic sequence generator is affected by the calculation precision of processor and quantization method, which leads to the quantized chaotic binary sequences emerging from some periodic phenomena in the local range of sequences. However, in view of the current methods for analyzing the periodicity and randomness of binary sequences, evaluating the periodic characteristics which exist in the local range of chaotic binary sequences is extremely difficult. Therefore, in this paper, we have proposed a novel algorithm called the GBSPD (General Binary Sequences Periodic Detection) algorithm, which can detect typical periodic phenomena and local periodic phenomena in the chaotic binary sequence. Moreover, it can accurately locate the position of periodic phenomena of binary sequences and perform a detailed statistical analysis. Furthermore, by using the GBSPD algorithm to detect the periodic characteristics of the quantified logistic chaotic binary sequences, the experimental results show that the algorithm can effectively locate multiple periodic phenomena in the binary sequences.
\end{abstract}

Keywords: Chaos; periodicity detection; binary sequences; randomness evaluation.

\section{Introduction}

Chaotic systems possess many good characteristics such as intrinsic stochasticity, initial value sensitivity and positive Lyapunov exponent so that these systems have been widely used in secret communication and encryption system. However, whether discrete time chaotic systems such as logistic and Henon or continuous time chaotic systems such as Lorenz Chen \& Ueta, 1999] and Sprott, 1994] converted these systems into chaotic binary sequences with the purpose of encrypting data, this needs to be quantified. In the process of quantization, the statistical properties and randomicity of chaotic systems will be affected. The calculation precision and quantization method are two important factors that degenerate the characteristics of chaotic system.

As early as 1989, Wheeler [1989] found that the chaotic cipher sequences existed in a short periodic phenomenon when analyzing the key space based on logistic mapping. In 1997. when Kohda and Tsuneda [1997] analyzed the statistical properties of chaotic binary sequences, it was found that the calculation precision had a significant influence on the randomness of chaotic sequences. Since then some references Persohn \& Povinelli, 2012;

\footnotetext{
${ }_{\ddagger}$ Author for correspondence

This is an Open Access article published by World Scientific Publishing Company. It is distributed under the terms of the Creative Commons Attribution 4.0 (CC-BY) License. Further distribution of this work is permitted, provided the original work is properly cited.
} 
Tsuneda et al., 2007; Sheng \& Quan, 2010; James, 1988; Bresten \& Jung. 2009: Hua et al. . 2018: Li et al., 2005] have also indicated that the computational precision and quantization method can degrade the performance of the chaotic system. This phenomenon makes the security of some encryption systems not guaranteed when using the local range of chaotic binary sequences Li et al., 2003]. At present, it is extremely difficult to prove whether a generator is a real random number generator. Therefore, some scholars will measure the statistical characteristics of random number generator by means of statistical test of randomness, such as ENT randomness test Walker, 2008], Diehard randomness test Brown, 2018], NIST-800-22 [Guinee \& Blaszczyk, 2009], TestU01 L'Ecuyer \& Simard, 2007], etc. These methods Shannon. 1948: Jessa \& Walentynowicz, 2001; Tsuneda, 2005; Candan et al., 1998 used Shannon entropy, sequence autocorrelation, accumulation and discrete Fourier transform to evaluate randomness of chaotic binary sequences. However, the stability of the sequence is often neglected while focusing on the randomness of the binary sequence. Some of the seemingly random chaotic sequences may have some local periodic phenomena in local range of sequences. From the point of view of encryption application, the stability of binary sequence has great influence on the security of encryption system. Based on the above considerations and analysis, the study of periodic phenomena in the local range of digital chaotic binary sequence has crucial significance for chaotic digital secure communication.

At present, the methods of detecting the periodic phenomena of binary sequences Zhang et al., 2015; Xiao et al., 2004; Sang et al., 2001; Elfeky et al., 2005] are mainly to analyze the precise period and approximate periodic phenomena of the sequences, such as power spectrum analysis, discrete Fourier transform, autocorrelation test, approximate entropy, permutation entropy, pattern mining and some analytical methods based on periodic template. Hasimoto-Beltrán and RamírezRamírez [2011] proposed an unrestricted search algorithm to track down the cycle of chaotic sequence with the purpose of eliminating short cycles. Viana et al. 2012] reported a periodicitydetection algorithm to evaluate the periodicity of experimental time series. Nevertheless, these methods cannot detect the local periodic phenomena of chaotic sequences. The above methods can only estimate whether there is a periodic phenomenon in the binary sequences from the global point of view when analyzing the periodic phenomenon. In addition to this, there are few studies on the potential periodic phenomena in the local range of sequences, and the precise positioning of periodic phenomena cannot be achieved. Furthermore, Zheng et al. 2012] designed a binary sequence period detection algorithm to evaluate the periodicity in a binary chaotic sequence. Although this algorithm can detect and locate many kinds of periodic phenomena, it cannot detect all local periodic phenomena of chaotic binary sequences. However, for GBSPD algorithm, not only can it detect all periodic phenomena, but it also can accurately locate all precise period, approximate period and local period positions of the chaotic binary sequence.

In view of the above problems, this paper firstly extends the definition of multiple periodic phenomena in chaotic binary sequence, including precise period, approximate period and local periodic phenomena. Furthermore, a novel GBSPD (General Binary Sequences Periodic Detection) algorithm was proposed, which can accurately estimate whether there are precise period, approximate period and local periodic phenomena for chaotic binary sequences by generating sequence reconstruction matrix and periodic detection matrix. In addition, it can accurately locate the position of the periodic template so as to effectively evaluate the randomness and security of the binary sequences.

\section{Analysis and Definition of Binary Sequence Periodic Phenomena}

\subsection{Local periodic phenomena of chaotic binary sequence}

For a binary sequence $\varepsilon(t)$, if $\varepsilon(t)$ satisfies the equation $\varepsilon(t)=\varepsilon(t+n T)$, this sequence has a periodic phenomenon with period length $T$. However, for some chaotic binary sequences, a particular periodic phenomenon is shown in Fig. 1. As can be seen from the figure, $L_{T}$ bits can appear as the same bit string of $\omega$ bits from a truncated fragment of the quantized chaotic binary sequence. In this paper, this bit string "110111001" is called the period template with length $\omega$. Nevertheless, the local nonrandom phenomena of binary sequence cannot be detected by some current randomness measurement methods, such as discrete Fourier transform, 


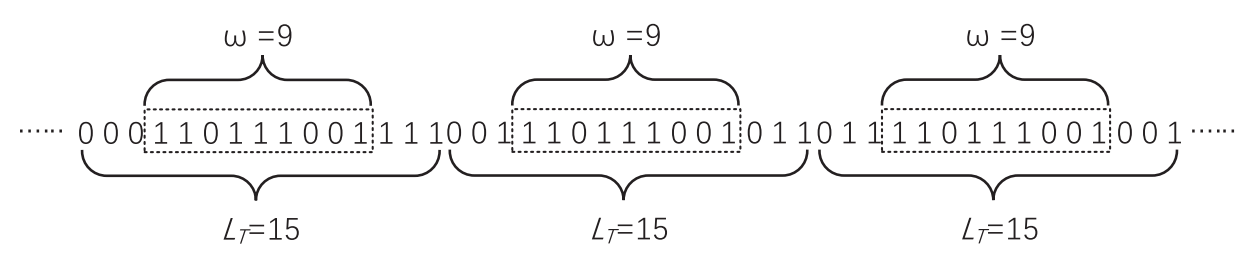

Fig. 1. Local periodic phenomena of chaotic binary sequence.

autocorrelation test, run test and random deviation test, etc. The occurrence of those phenomena will inevitably reduce the random performance of the chaotic binary sequence. If the sequence is used as the key stream of the stream cipher, the anti-attack performance of ciphertext will be seriously affected. Therefore, the detection and accurate localization of the above phenomena is of great significance to evaluate the randomness performance of chaotic binary sequence.

\subsection{Extended definition of periodic phenomena}

For a continuous positive integer set $\left\{\phi_{\alpha} \mid 1 \leq \alpha \leq\right.$ $\left.(\omega+\alpha-1) \leq L_{T}\right\}$, if arbitrary integer $\alpha$ can satisfy Eq. (1), the binary sequence $\varepsilon(t)$ has a generalized periodic phenomenon with period length $L_{T}$ in the interval $\left[L_{1}, L_{n}\right]$

$$
\varepsilon\left(L_{1}-1+\alpha\right)=\varepsilon\left(L_{1}-1+(k-1) * L_{T}+\alpha\right),
$$

where integer $k \in\left[1,\left(L_{n}-L_{1}+1\right) / L_{T}\right]$. The symbol string $\varepsilon\left(L_{1}-1+\alpha\right) \varepsilon\left(L_{1}-1+(\alpha+1)\right) \cdots \varepsilon\left(L_{1}-\right.$ $1+(\omega+\alpha-1))$ is called the period template, and its length is $\omega$.

(1) If $L_{1}=1$ and $L_{n} \rightarrow+\infty, \alpha$ is a continuous positive integer in the interval $\left[1, L_{T}\right]$, Eq. (1) describes a precise periodic phenomenon with the period length $L_{T}$. In this case, $\omega=L_{T}$.

(2) If $L_{1} \rightarrow 1, L_{n} \rightarrow+\infty$ and $\omega \rightarrow L_{T}$, Eq. (11) describes an approximate periodic phenomenon with the period length $L_{T}$.

(3) If interval $\left[L_{1}, L_{n}\right]$ is a local interval of the binary sequence, and $0<\omega<L_{T}$, Eq. (1) describes a local periodic phenomenon.

\section{The Implementation of GBSPD Algorithm}

For arbitrary binary sequence $\varepsilon(t)$ with length $n$, a $(n-1) *(n-1)$ matrix $R$ is defined as sequence reconstruction matrix. The matrix $R$ satisfies equation $R_{i, j}=\varepsilon(i+1) \odot \varepsilon(j)$, which can be given as a lower triangular matrix.

$R=\left[\begin{array}{ccccc}R_{1,1} & & & & \\ R_{2,1} & R_{2,2} & & & \\ \vdots & \vdots & \ddots & & \\ R_{n-2,1} & R_{n-2,2} & \cdots & R_{n-2, n-2} & \\ R_{n-1,1} & R_{n-1,2} & \cdots & R_{n-1, n-2} & R_{n-1, n-1}\end{array}\right]$,

$R_{i, j}=\varepsilon(i+1) \odot \varepsilon(j)$.

If $\varepsilon(t)$ satisfies $\varepsilon(i) \varepsilon(i+1) \cdots \varepsilon(i+\omega-1)=\varepsilon(i+$ $j) \varepsilon(i+j+1) \cdots \varepsilon(i+j+\omega-1)$ there are $\omega$ consecutive elements of equality from the beginning of $\varepsilon(i)$ and $\varepsilon(i+j)$. Further, the matrix $R$ can also reflect the above relationship. Specifically, there is a run of ones with length $\omega$ between the $i$ th and $(i+\omega-1)$ th elements of the $j$ th diagonal line of matrix $R$.

For example, we assume that $\varepsilon(t)=$ "1011011", and matrix $Q$ is a $(n-1) *(n-1)$ upper triangulation matrix. $Q_{i, j}=\omega$ can represent $\varepsilon(i) \varepsilon(i+1) \cdots \varepsilon(i+$ $\omega-1)=\varepsilon(i+j) \varepsilon(i+j+1) \cdots \varepsilon(i+j+\omega-1)$. According to the above definition, matrix $Q$ and sequence reconstruction matrix $R$ can be shown below:

$$
\begin{aligned}
R & =\left[\begin{array}{lllllll}
0 & & & & & \\
1 & 0 & & & & \\
1 & 0 & 1 & & & \\
0 & 1 & 0 & 0 & & \\
1 & 0 & 1 & 1 & 0 & \\
1 & 0 & 1 & 1 & 0 & 1
\end{array}\right], \\
Q & =\left[\begin{array}{llllll}
0 & 1 & 4 & 0 & 1 & 1 \\
0 & 0 & 3 & 0 & 0 & \\
1 & 0 & 2 & 1 & & \\
0 & 1 & 1 & & & \\
0 & 0 & & & \\
1 & &
\end{array}\right] .
\end{aligned}
$$


As can be seen from Eqs. (3) and (4), there is a definite correspondence between matrix $Q$ and sequence reconstruction matrix $R$. For example, $Q_{1,3}=4$ indicates that the run of ones with four elements can be found at the beginning of the first element of the third diagonal line of matrix $R$. In a similar way, $Q_{i, j}=\omega$ indicates that the run of ones with $\omega$ elements can be found at the beginning of the $i$ th element of the $j$ th diagonal line of matrix $R$. The above phenomenon shows that the position of the period template of the binary sequence $\varepsilon(t)$ has a clear correspondence with the sequence reconstruction matrix $R$.

In this section, we assume that $S_{k}(t)$ is the $k$ th diagonal line of matrix $R$. $L_{k}$ represents the length of $S_{k}(t) . S_{k}^{i}$ is the $i$ th element value of $S_{k}(t)$, and $1 \leq i \leq L_{k}$. The period detection matrix $G$ with $r$ row, $k$ column is generated by folding the sequence $S_{k}(t)$ according to each $k$ element, where $k \in[2, n / 2]$, and $r$ is given as:

$$
r= \begin{cases}\frac{L_{k}}{k}, & L_{k} \bmod k=0, \\ \text { floor }\left(\frac{L_{k}}{k}\right), & L_{k} \bmod k>0 .\end{cases}
$$

For Eq. (5), floor() function represents round down. Moreover, period detection matrix $G$ can be expressed as follows:

$$
\begin{aligned}
& G=\left[\begin{array}{cccc}
G_{1,1} & G_{1,2} & \cdots & G_{1, k} \\
G_{2,1} & G_{2,2} & \cdots & G_{2, k} \\
\vdots & \vdots & & \vdots \\
G_{r, 1} & G_{r, 2} & \cdots & G_{r, k}
\end{array}\right] \\
& =\left[\begin{array}{cccc}
S_{k}^{1} & S_{k}^{2} & \cdots & S_{k}^{k} \\
S_{k}^{k+1} & S_{k}^{k+2} & \cdots & S_{k}^{2 k} \\
\vdots & \vdots & & \vdots \\
S_{k}^{(r-1) * k+1} & S_{k}^{(r-1) * k+2} & \cdots & S_{k}^{r * k}
\end{array}\right] .
\end{aligned}
$$

\subsection{Detection and location of precise period}

One-dimensional row vector

$$
V=\left[\begin{array}{lllll}
V_{1}^{2}, & \cdots & V_{\text {index }}^{k}, \quad \cdots & V_{(n / 2-2)+1}^{n / 2}
\end{array}\right]
$$

is constructed and $V_{\text {index }}^{k}$ is defined as:

$$
V_{\text {index }}^{k}=\prod_{i=1}^{r * k} S_{k}^{i}, \quad k \in\left[2, \frac{n}{2}\right] .
$$

If all elements of vector $V$ are zero, the binary sequence $\varepsilon(t)$ does not have precise periodic phenomenon. On the contrary, for vector $V$, if there are one or more elements which are equal to one, the binary sequence $\varepsilon(t)$ has precise periodic phenomenon. The first element $V_{\text {index }}^{k}$ is to be found which is equal to one in ascending order of the vector $V$, and the superscript $k$ of the $V_{\text {index }}^{k}$ is the period length of $\varepsilon(t)$.

\subsection{Detection and location of approximate period}

One-dimensional row vector

$$
T=[T(1), \quad \cdots \quad T(m), \quad \cdots \quad T(k)]
$$

is constructed and $T(m)$ is defined as:

$$
T(m)=\prod_{i=0}^{r-1} S_{k}^{i * k+m}, \quad 1 \leq m \leq k,
$$

where the value range of $T(m)$ is $\{0,1\}$. The start and end points of the longest run of ones of vector $T$ are searched by the location algorithm of longest run of ones. This algorithm is given in Algorithm 1 as a pseudo code.

Based on the above-mentioned pseudo code, the start and end points of the longest run of ones of vector $T$ can be accurately located. We assume that the start and end points of the longest run of ones are $t$ Start and $t$ End, respectively. Its length is $r$ Len. If equation $r L e n / k>$ threshold $_{1}$ is satisfied, the binary sequence $\varepsilon(t)$ appears with the approximate periodic phenomena, where threshold 1 represents the decision threshold of approximate period, and the value range of threshold 1 is $(0,1)$. The larger the threshold ${ }_{1}$ is, the more obvious the approximate periodic phenomenon of $\varepsilon(t)$ is. The position of the approximate period can be given as:

$$
\begin{aligned}
& \varepsilon(t \text { Start }) \cdots \varepsilon(t \text { End }) \\
& =\varepsilon(t \text { Start }+i * k) \cdots \varepsilon(t \text { End }+i * k), \\
& 1 \leq i \leq(r-1) .
\end{aligned}
$$




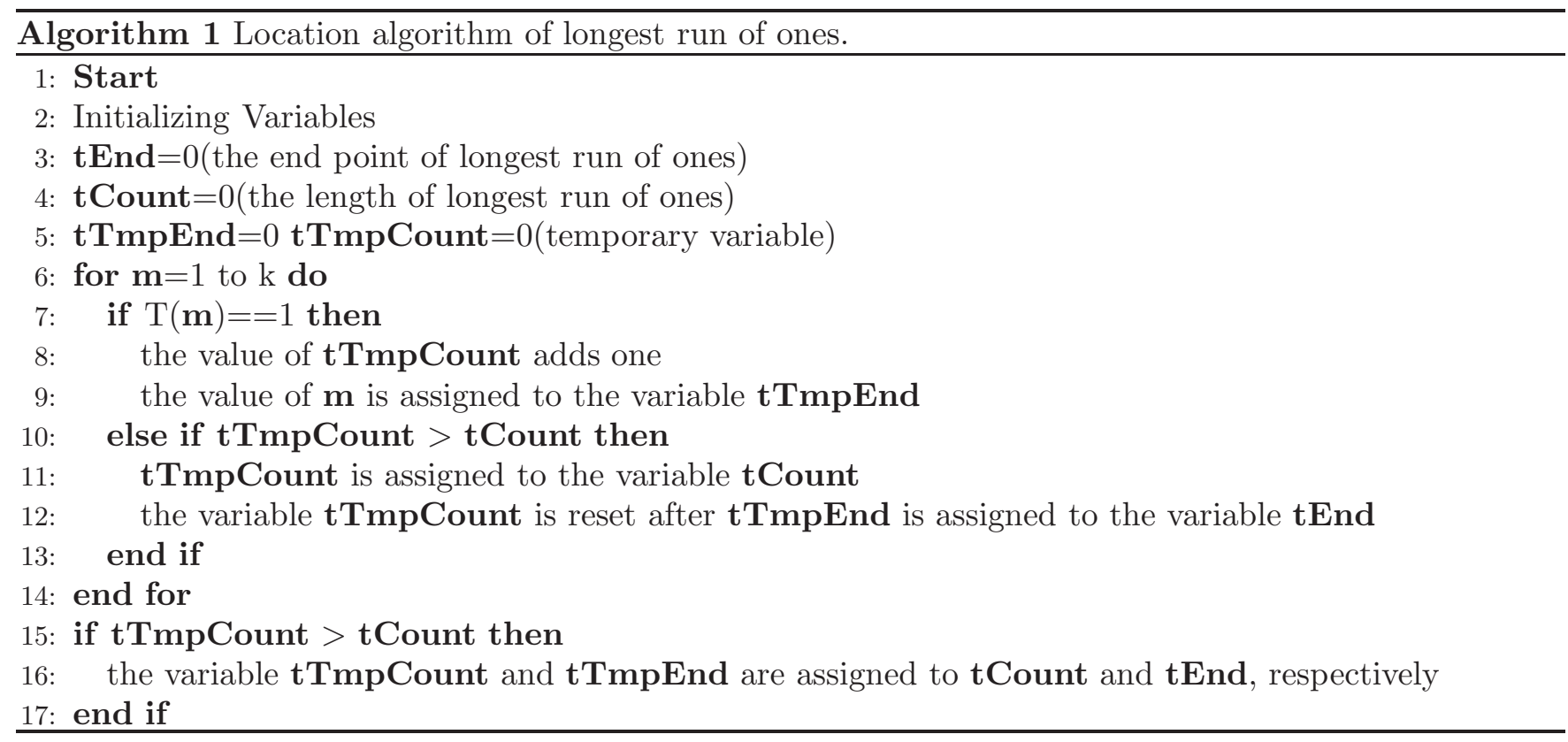

\subsection{Detection and location of local period}

Firstly, a matrix group $J$ is constructed and defined as follows:

$$
\begin{aligned}
J & =\left[\begin{array}{lllll}
J_{2}, & \cdots & J_{x}, & \cdots & J_{(r-1)}
\end{array}\right], \quad x \in[2, r-1], \\
J_{x} & =\left[\begin{array}{lllll}
J_{x}(1), & \cdots & J_{x}(i), & \cdots & J_{x}(r-x+1)
\end{array}\right], \quad i \in[1, r-x+1],
\end{aligned}
$$

where the submatrix $J_{x}(i)$ is extracted from the periodic detection matrix $G$, and $J_{x}(i)$ is defined as:

$$
J_{x}(i)=\left[\begin{array}{cccc}
S_{k}^{(i-1) * k+1} & S_{k}^{(i-1) * k+2} & \cdots & S_{k}^{i * k} \\
S_{k}^{i * k+1} & S_{k}^{i * k+2} & \cdots & S_{k}^{(i+1) * k} \\
\vdots & \vdots & & \vdots \\
S_{k}^{(x+i-2) * k+1} & S_{k}^{(x+i-2) * k+2} & \cdots & S_{k}^{(x+i-1) * k}
\end{array}\right] .
$$

Secondly, the one-dimensional row vector $Y$ is given as follows:

$$
\begin{gathered}
Y=\left[\begin{array}{llll}
Y(1), \quad \cdots & Y(j), \quad \cdots & Y(k)
\end{array}\right], \quad j \in[1, k] \\
Y(j)=\prod_{m=0}^{x-1} S_{k}^{(i-1+m) * k+j},
\end{gathered}
$$

where the value range of $Y(j)$ is $\{0,1\}$. For onedimensional row vector $Y$, the start and end points of the longest run of ones are searched by the location algorithm of longest run of ones. We assume that the start and end points of the longest run of ones are $t$ Start and $t$ End, respectively. Moreover, the length of the longest run of ones is rLen. If equation $r \operatorname{Len} *(x+1) / n \geq$ threshold $_{2}$ is satisfied, the binary sequence $\varepsilon(t)$ has the local periodic phenomena, where threshold ${ }_{2}$ represents the decision threshold of local period, and the value range of threshold $_{2}$ is $(0,1)$. The larger the threshold 2 is, the more obvious the local periodic phenomenon of binary sequence is. Further, the precise location of local period of the binary sequence can be given as:

$$
\begin{aligned}
& \varepsilon((i-1) * k+t \text { Start }) \cdots \varepsilon((i-1) * k+t \text { End }) \\
&= \varepsilon((i-1+l) * k+t \text { Start }) \\
& \cdots \varepsilon((i-1+l) * k+t \text { End }), \quad l \in[1, x] .
\end{aligned}
$$




\section{The Periodic Phenomena Detection of Logistic Chaotic Mapping}

In this paper, we generated the chaotic binary sequences for the experiment by means of logistic chaotic mapping and the corresponding quantization methods. The logistic system equation May, 1976] can be described in the following iterative form:

$$
x_{n+1}=\mu x_{n}\left(1-x_{n}\right), \quad \mu \in(0,4], \quad x_{n} \in(0,1)
$$

where $\mu$ is called branch parameter, when the value range of $\mu$ is [3.5699456, 4], the logistic mapping is in a chaotic state and displays complex dynamic characteristics. Further, the corresponding quantization method is defined as follows:

$$
s_{n}= \begin{cases}0, & x_{n}<c \\ 1, & x_{n} \geq c\end{cases}
$$

where $c=0.5,\left\{x_{n}\right\}$ and $\left\{s_{n}\right\}$ represent the chaotic real value sequence and the quantized chaotic binary sequence, respectively. The chaotic real value sequence can be converted into chaotic binary sequence by Eq. (15). The iterative precision $M=16,24$ and 32 are respectively adopted to generate different chaotic binary sequences. Moreover, for some current randomness detection method of binary sequence, autocorrelation test and multiscale permutation entropy are performed in order to compare with the GBSPD algorithm. As can be seen from the experimental results, not only can the GBSPD algorithm detect the periodic phenomena but also it can accurately locate the precise period, approximate period and local period position of the chaotic binary sequence.

\subsection{Autocorrelation test}

Autocorrelation can clearly reflect the dependence relationship of a signal between two different moments, which is an important method with the purpose of detecting randomness and periodicity of the binary sequence. Autocorrelation function is defined as follows:

$$
R_{s}(m)=\sum_{n=-\infty}^{\infty} s(n) s(n+m),
$$

where $s(n)$ and $R_{s}(m)$ denote chaotic binary sequence and autocorrelation function, respectively.
By using the above equation, the autocorrelation test can be performed with logistic binary sequences of different iterative precision. MATLAB simulation results are shown in Fig. 2.

As can be seen from Fig. 2, when the iteration precision $M=16$, Fig. 2(a) displays a dense peak line with an equal amplitude. In this case, the logistic binary sequence shows the obvious periodicity. When the iteration precision $M=24$, the amplitude of the peak line decreases gradually, and it shows an approximate periodic phenomenon. Finally, when the iteration precision $M=32$, the autocorrelation function has only one peak line. Figure 2(c) indicates that the logistic binary sequence with $M=32$ has good randomness.

\subsection{Multiscale permutation entropy test}

Multiscale permutation entropy (MPE) [Bandt \& Pompe, 2002; [Niu \& Zhang, 2017] is widely applied in the measurement of binary sequence complexity and nonlinear analysis because of its high robustness and fast and simple algorithm characteristics, which can be described as follows:

For a binary sequence $x_{L}=\left\{x_{1}, x_{2}, \ldots, x_{L}\right\}$ with length $L$, its coarse-grained process is shown in the following equation:

$$
y_{j}^{s}=\frac{1}{s} \sum_{i=(j-1) s+1}^{j s} x_{i}, \quad 1 \leq j \leq \frac{L}{s},
$$

where $s$ and $y_{j}^{s}$ represent scale factor and the binary sequences of different scale, respectively. Then, the sequence $y_{j}^{s}$ is reconstructed as:

$$
\begin{array}{r}
Y_{t}^{s}=\left\{y_{t}^{s}, y_{t+\tau}^{s}, \ldots, y_{t+(m-1) \tau}^{s}\right\}, \\
t \in\left[1, \frac{L}{s}-m+1\right],
\end{array}
$$

where $m$ and $\tau$ denote embedding dimension and delay factor, respectively. The reconstructed sequence $Y_{t}^{s}$ is placed in an ascending order:

$$
y_{t+\left(k_{1}-1\right) \tau}^{s} \leq y_{t+\left(k_{2}-1\right) \tau}^{s} \leq \cdots \leq y_{t+\left(k_{m}-1\right) \tau}^{s} .
$$

For reconstructed sequence $Y_{t}^{s}, \pi_{t}=\left\{k_{1}, k_{2}, \ldots\right.$, $\left.k_{m}\right\}$ displays the original position index of each element, which is one of the possible order types of all $m$ ! permutations. Further, $N_{l}$ represents the number of order types of each permutation, and its 


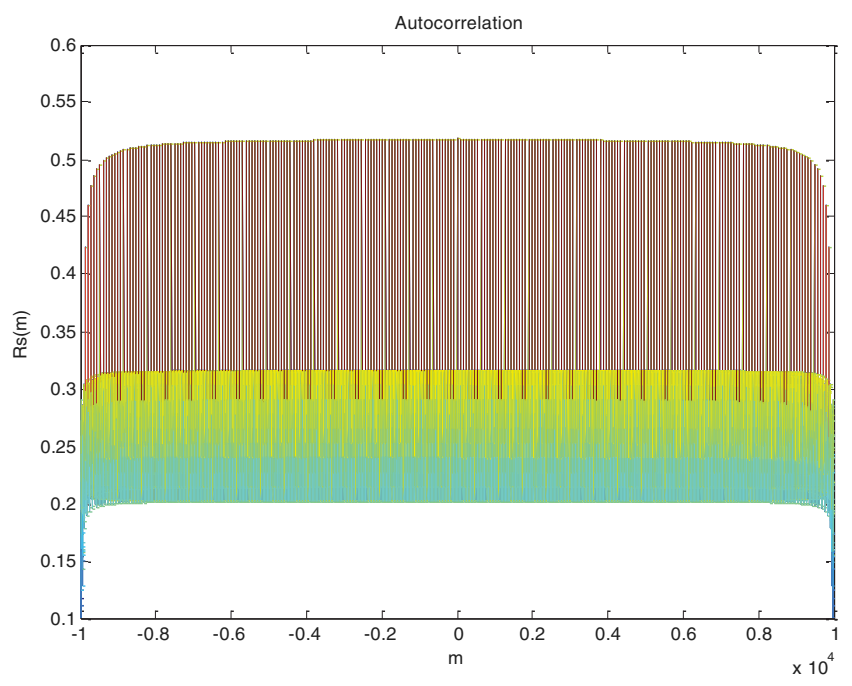

(a)

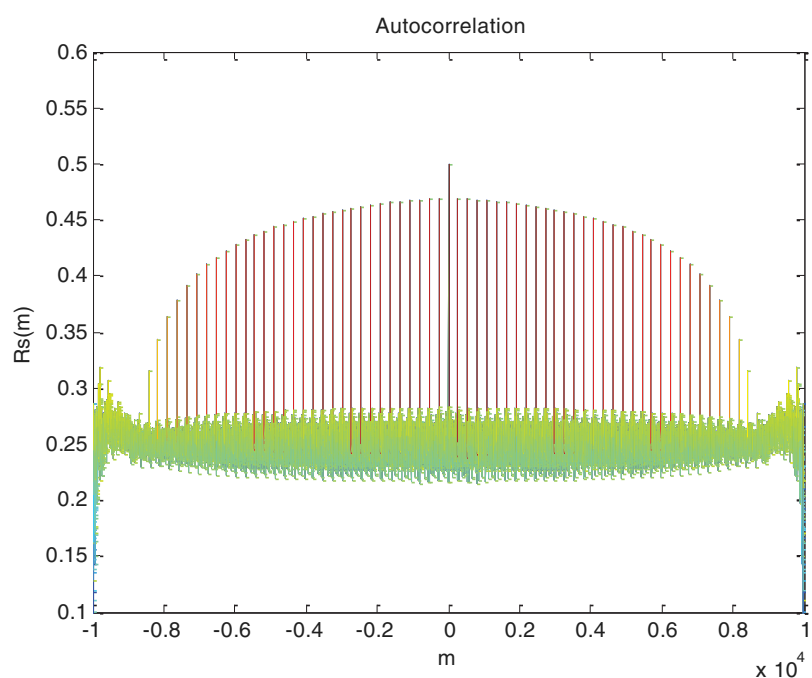

(b)

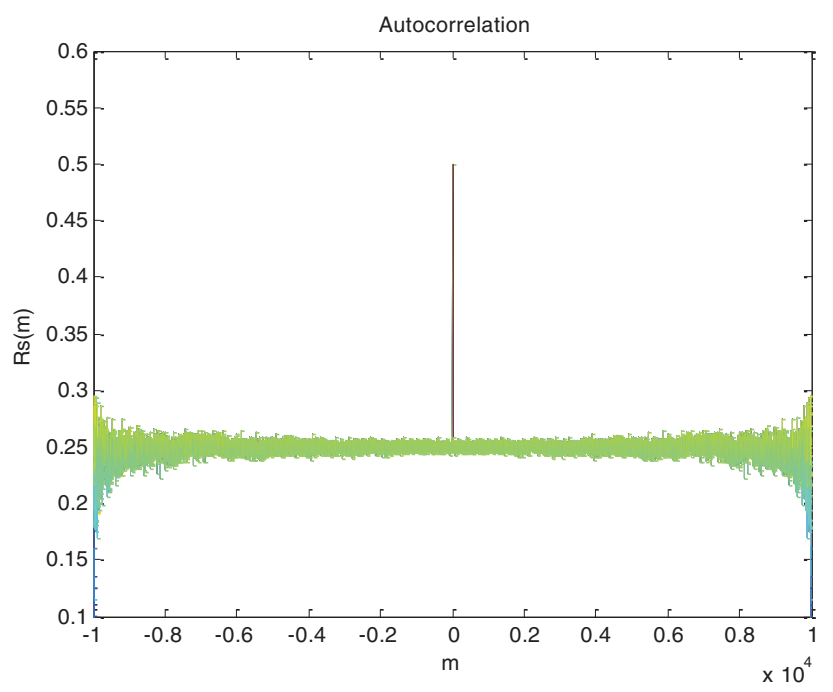

(c)

Fig. 2. Autocorrelation test of logistic binary sequence: (a) $M=16$, (b) $M=24$ and (c) $M=32$.

probability is shown in the following equation:

$$
P_{l}^{s}=\frac{N_{l}}{\frac{L}{s}-m+1} .
$$

Thus, MPE with scale factor $s$ can be defined as follows:

$$
H_{P}^{s}=-\sum_{l=1}^{m !} P_{l}^{s} \ln P_{l}^{s}
$$

when $P_{l}^{s}=1 / m$ !, $H_{P}^{s}$ obtains the maximum value $\ln (m !)$. Further, the normalized MPE can be defined as $h_{P}^{s}=H_{P}^{s} / \ln (m !)$.
MPE test can be performed with logistic binary sequences of different iterative precision. We respectively chose embedding dimension $m=6$, delay factor $\tau=1$ and scale factor $s \in[5,20]$ as the parameters of MPE. MATLAB simulation results are shown in Fig. 3 ,

As can be seen from Fig. 3, when the iteration precision $M=32$, the logistic binary sequence has the largest MPE value, which can display a good complexity. The MPE value of logistic binary sequence with $M=24$ is higher than that with $M=16$. Based on the above analysis, we can come to a conclusion that MPE value of the sequence can reflect the randomness and complexity of the 


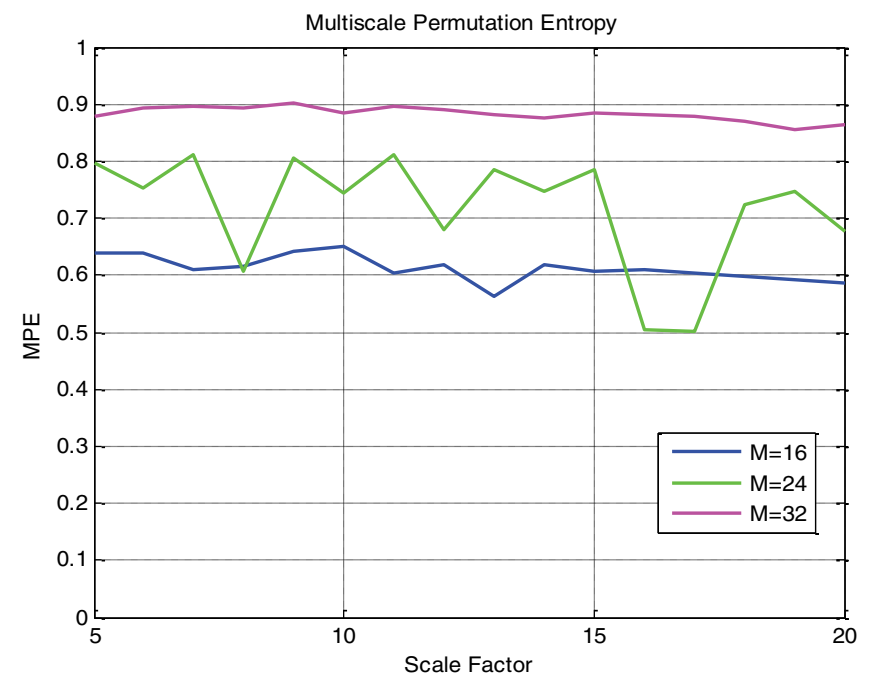

Fig. 3. MPE of the logistic binary sequence.

Table 1. ApEn values of the logistic binary sequences with different iteration precision.

\begin{tabular}{cc}
\hline Iteration Precision $M$ & ApEn Value \\
\hline 16 & 0.6408 \\
24 & 0.6717 \\
32 & 0.6918 \\
\hline
\end{tabular}

sequence to a certain extent. However, this method cannot accurately detect the periodic phenomena of the binary sequence.

\subsection{Approximate entropy test}

Approximate entropy (ApEn) was proposed by Pincus [1991], which can serve as a measure of sequence complexity. In this experiment, we calculated the ApEn of logistic binary sequences with different iteration precision. The parameters of ApEn have embedding dimension $m$ and similar tolerance $r$. In this section, we set $m=2$ and $r=0.1$. The experimental results are shown in Table 1 As can be seen from the table, the logistic binary sequence with $M=32$ has the maximum entropy value. However, this method can only evaluate the complexity of the different binary sequences, and cannot measure the local periodic phenomena of the sequence.

\subsection{GBSPD algorithm}

In this section, GBSPD test can be performed with logistic binary sequence of different iterative precision, and the length of sequence is 1000. GBSPD algorithm is implemented through MATLAB programs with the purpose of evaluating the performance of chaotic binary sequence, the experimental results are shown in Fig. 4. As can be seen from Figs. 4(a)-4(c), when the iteration precision $M=16$, the logistic binary sequence appears as a precise periodic phenomenon and period length is $L_{T}=79$. When the iteration precision $M=24$, the logistic binary sequence has an approximate periodic phenomenon. Moreover, the length of period template $\omega$ and period length $L_{T}$ are 247 and 272, respectively. The position of period template can be precisely located between the fifth column and 251st column. When the iteration precision $M=32$, the logistic binary sequence appears as a local periodic phenomenon. The length of period template $\omega$ and period length $L_{T}$ are 9 and 166, respectively. Further, the period template $\omega=$ "110111001" was localized between fourth row, 130th column and sixth row, 138th column.

Further, to demonstrate the effectiveness of this method, Tent binary sequences were generated by the same quantitative method and the length of sequence is 1000 . We generate three different Tent binary sequences with iteration precision $M=8$, 16 and 32. GBSPD algorithm is implemented to detect the periodic phenomena of binary sequence.

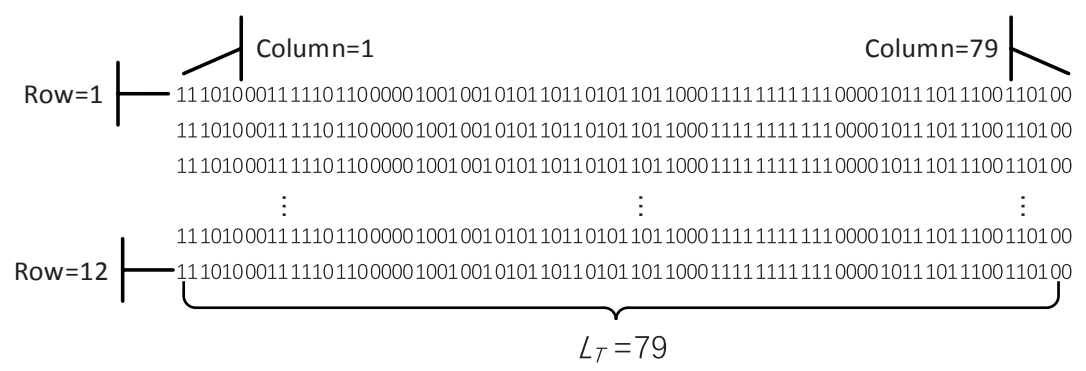

(a)

Fig. 4. The experimental results of GBSPD test for the logistic binary sequence: (a) $M=16$, (b) $M=24$ and (c) $M=32$. 


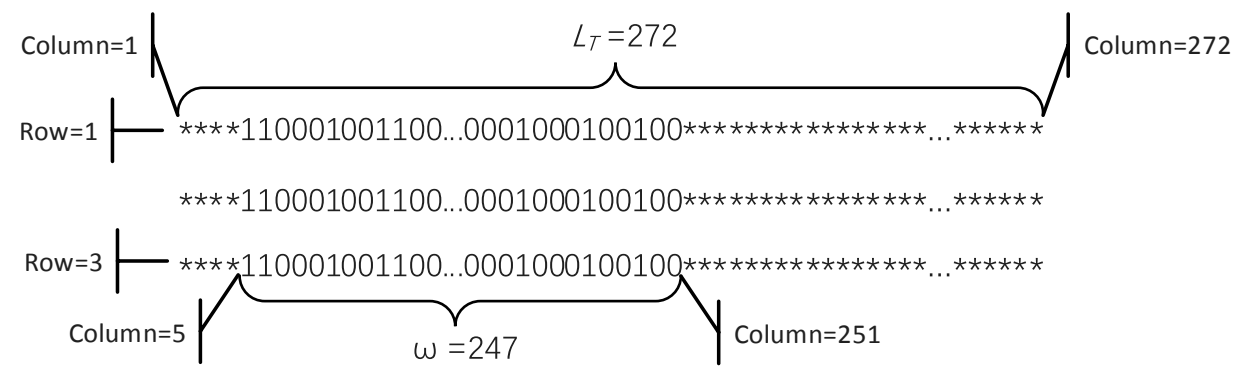

(b)

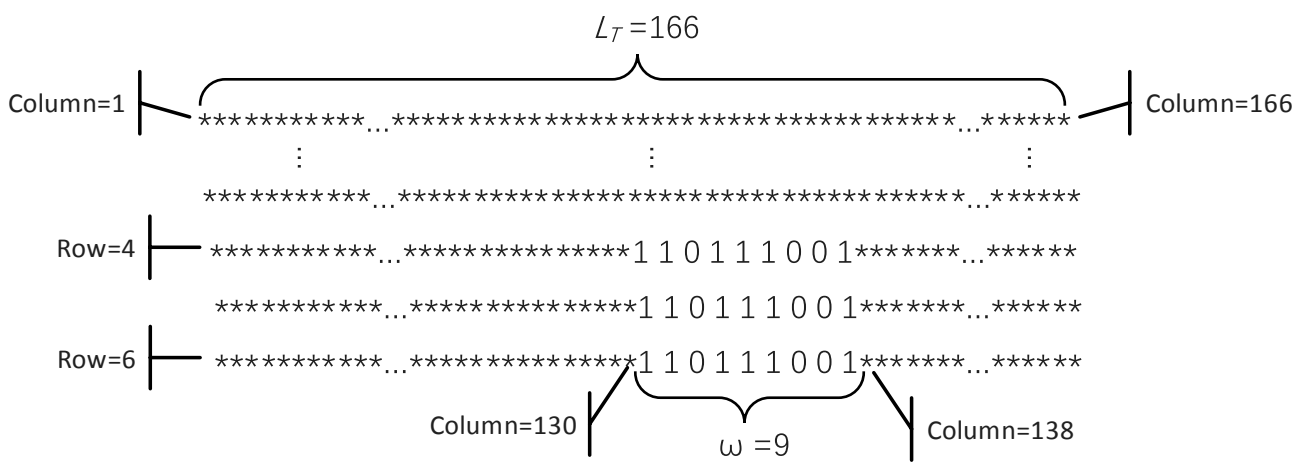

(c)

Fig. 4. (Continued)

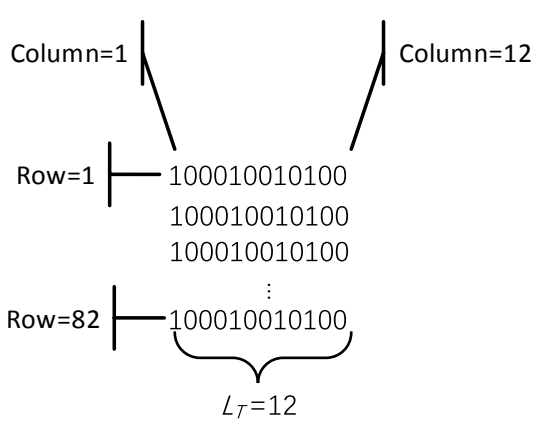

(a)

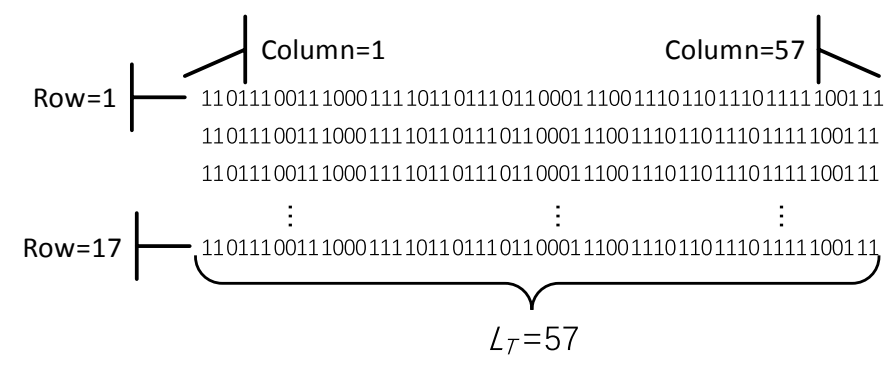

(b)

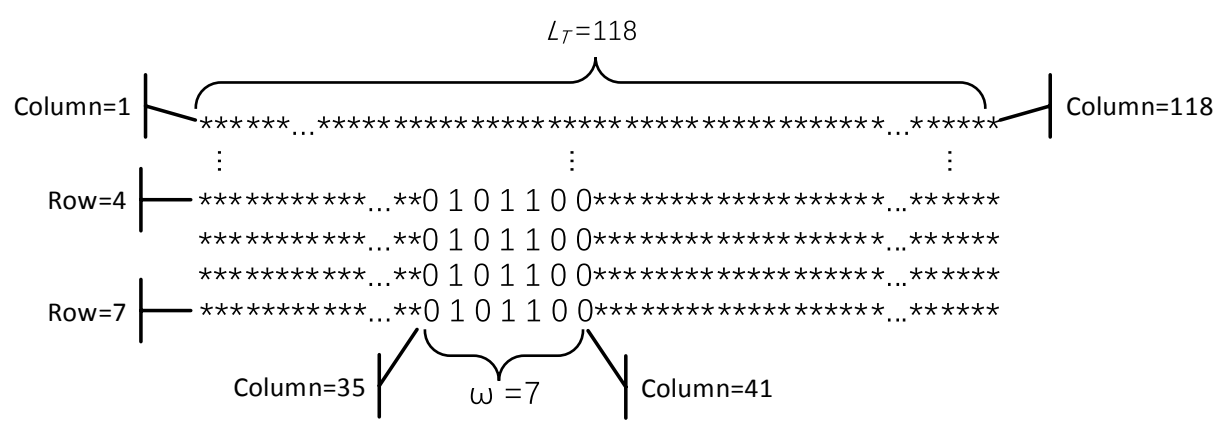

(c)

Fig. 5. The experimental results of GBSPD test for Tent binary sequence: (a) $M=8$, (b) $M=16$ and (c) $M=32$. 
The experimental results are shown in Fig. 5. As can be seen from Figs. 5(a)-5(c), when the iteration precision $M=8$ and 16 , the precise periodic phenomenon was detected by GBSPD algorithm, and the period length $L_{T}$ of the two sequences are equal to 12 and 57, respectively. When the iteration precision $M=32$, Tent binary sequence shows a local periodic phenomenon. The length of period template $\omega$ and period length $L_{T}$ are 7 and 118, respectively. The period template $\omega=$ "0101100" was localized between fourth row, 35th column and seventh row, 41st column. Based on the above experimental results, not only can the GBSPD algorithm detect the periodic phenomena, but also it can accurately locate the precise period, approximate period and local period position of the chaotic binary sequences.

\section{Conclusions}

In this paper, we described the quantized chaotic binary sequences to have some periodic phenomena in the local range of sequences. Then we extended the definition of period based on this phenomenon. In addition, we proposed a GBSPD algorithm which can accurately detect the existence of precise period, approximate period and local period in chaotic binary sequence. Meanwhile, the extraction method of the period template was described in detail, and multiple periodic phenomena in the chaotic binary sequences can be accurately searched for by this algorithm. Finally, the logistic binary sequences with different iteration precision were generated as experimental data. As can be seen from the experimental results, GBSPD algorithm can effectively evaluate and locate multiple periodic phenomena in comparison with autocorrelation and MPE algorithm. Furthermore, the implementation of GBSPD algorithm is significant for evaluating the randomness and security of chaotic binary sequences.

\section{Acknowledgments}

This work was supported by Natural Science Foundation of China (No. 61471158) and Postgraduate Innovation Program of Heilongjiang University (YJSCX2019-016HLTU).

\section{References}

Bandt, C. \& Pompe, B. [2002] "Permutation entropy: A natural complexity measure for time series," Phys. Rev. Lett. 88, 174102.

Bresten, C. L. \& Jung, J. H. [2009] "A study on the numerical convergence of the discrete logistic map," Commun. Nonlin. Sci. Numer. Simulat. 14, 30763088.

Brown, R. [2018] "Dieharder: A random number test suite, version 3.31.1," http://webhome.phy.duke. $\mathrm{edu} /{ }^{\sim} \mathrm{rgb} /$ General/dieharder.php.

Candan, C., Kutay, M. A. \& Ozaktas, H. M. [1998] "The discrete fractional Fourier transform," IEEE Trans. Sign. Process. 48, 1329-1337.

Chen, G. R. \& Ueta, T. [1999] "Yet another chaotic attractor," Int. J. Bifurcation and Chaos 9, 14651466.

Elfeky, M. G., Aref, W. G. \& Elmagarmid, A. K. [2005] "Periodicity detection in time series databases," IEEE Trans. Knowl. Data Engin. 17, 875-887.

Guinee, R. A. \& Blaszczyk, M. [2009] "A novel true random binary sequence generator based on a chaotic double scroll oscillator combination with a pseudo random generator for cryptographic applications," Int. Conf. Internet Technology and Secured Transactions (London, UK), pp. 1-6.

Hasimoto-Beltrán, R. \& Ramírez-Ramírez, R. [2011] "Cycle detection for secure chaos-based encryption," Commun. Nonlin. Sci. Numer. Simulat. 16, 32033211.

Hua, Z. Y., Zhou, B. H. \& Zhou, Y. C. [2018] "Sine-transform-based chaotic system with FPGA implementation," IEEE Trans. Indust. Electron. 65, 2557-2566.

James, F. [1988] "A review of pseudorandom number generators," Comput. Phys. Commun. 6, 329344.

Jessa, M. \& Walentynowicz, M. [2001] "Statistical properties of number sequences generated by $1 \mathrm{D}$ chaotic maps considered as a potential source of pseudorandom number sequences," IEEE Int. Conf. Electronics (Valletta, Malta), pp. 449-455.

Kohda, T. \& Tsuneda, A. [1997] "Statistics of chaotic binary sequences," IEEE Trans. Inform. Th. 43, 104112.

L'Ecuyer, P. \& Simard, R. [2007] "TestU01: A C library for empirical testing of random number generators," ACM Trans. Math. Softw. 33, 1-40.

Li, S., Mou, X., Cai, Y., Ji, Z. \& Zhang, J. [2003] "On the security of a chaotic encryption scheme: Problems with computerized chaos in finite computing precision," Comput. Phys. Commun. 153, 52-58. 
Li, S. J., Chen, G. R. \& Mou, X. Q. [2005] "On the dynamical degradation of digital piecewise linear chaotic maps," Int. J. Bifurcation and Chaos 15,329344.

May, R. M. [1976] "Simple mathematical models with very complicated dynamics," Nature 261, 459-467.

Niu, H. \& Zhang, L. [2017] "Nonlinear multiscale entropy and recurrence quantification analysis of foreign exchange markets efficiency," Entropy 20, 1-16.

Persohn, K. J. \& Povinelli, R. J. [2012] "Analyzing logistic map pseudorandom number generators for periodicity induced by finite precision floating-point representation," Chaos Solit. Fract. 45, 238-245.

Pincus, S. M. [1991] "Approximate entropy as a measure of system complexity," Proc. Natl. Acad. Sci. USA 88, 2297-2301.

Sang, T., Wang, R. \& Yan, Y. [2001] "Generating binary Bernoulli sequences based on a class of evensymmetric chaotic maps," IEEE Trans. Commun. 49, 620-623.

Shannon, C. E. [1948] "A mathematical theory of communication," Bell Syst. Tech. J. 27, 379-423.

Sheng, L. Y. \& Quan, J. B. [2010] "Research on periods of chaotic sequences under computer iteration," J. Comput. Appl. 30, 1802-1804.

Sprott, J. C. [1994] "Some simple chaotic flows," Phys. Rev. E 50, R647-R650.

Tsuneda, A. [2005] "Design of binary sequences with tunable exponential autocorrelations and run statistics based on one-dimensional chaotic maps," IEEE Trans. Circuits Syst.-I: Reg. Papers 52, 454462.

Tsuneda, A., Mitsuishi, S. \& Inoue, T. [2007] "A study on random bit generators with post-processing by shift registers and modulo-2 addition," Eur. Conf. Circuit Theory and Design (Seville, Spain), pp. 631634.

Viana, E. R., Rubinger, R. M., Albuquerque, H. A., Dias, F. O., Oliveira, A. G. \& Ribeiro, G. M. [2012] "Periodicity detection on the parameter-space of a forced Chua's circuit," Nonlin. Dyn. 67, 385-392.

Walker, J. [2008] "ENT," http://www.fourmilab.ch/ random/.

Wheeler, D. [1989] "Problem with chaotic cryptosystems," Cryptologia 13, 243-250.

Xiao, F. H., Yan, G. R. \& Han, Y. H. [2004] "A symbolic dynamics approach for the complexity analysis of chaotic pseudorandom sequences," Acta Phys. Sin. 53, 2877-2881.

Zhang, L., Chen, S. \& Zhang, Y. [2015] "Time sequence mining algorithm based on autocorrelated period obtaining," J. Inform. Engin. Univ. 16, 209-213.

Zheng, Y. B., Song, Y., Du, B. X., Pan, J. \& Ding, Q. [2012] "A novel detection of periodic phenomena of binary chaotic sequences," Acta Phys. Sin. 61, 230501. 